\title{
Technological Prototypes and Result Highlights of Highly Granular Calorimeters
}

\author{
Gérald Grenier ${ }^{* \dagger}$ \\ Université Lyon 1, CNRS/IN2P3, IPN-Lyon, F-69622, Villeurbanne, France \\ E-mail: greniereipnl.in2p3.fr
}

The CALICE collaboration is developing highly granular calorimeters optimized for Particle Flow Algorithms (PFA). After having demonstrated the potential of these calorimeters in a first set of prototypes, a next generation of prototypes is under development that addresses full system requirements such as compactness and low power consumption. Improvements on the analysis and reconstruction of electromagnetic and hadronic showers is discussed along with tests on data of the performance of the Particle Flow Paradigm.

The European Physical Society Conference on High Energy Physics

5-12 July

Venice, Italy

${ }^{*}$ Speaker.

${ }^{\dagger}$ On behalf of CALICE collaboration. 


\section{Introduction}

Future $e^{+} e^{-}$collider projects, like ILC[1] or CEPC[2] require detectors with the ability to discriminate in mass $W$ and $Z$ in hadronic decays. To achieve that, a resolution in jet energy of about 3 to $4 \%$ or better is needed, which translate, at ILC energies, to a jet resolution stochastic term of $30 \%$ or better[3][4]. To achieve such a jet energy resolution, a promising way is to use the Particle Flow (PF) paradigm consisting in measuring the jet energy by summing the energy of the jet's charged particles energy measured in the tracker, the jet's photons energy measured in the electromagnetic calorimeter (ECAL) and the jet's neutral hadrons energy measured in the hadronic calorimeter (HCAL) [3][5]. To use the PF paradigm, detectors need to be able to separate energy depositions from close-by particles. This separation can be achieved with high granularity calorimeters. As an example, the baseline design of the ILD[4] detector has an ECAL with transverse cell size of $5 \times 5 \mathrm{~mm}^{2}$ and a HCAL transverse cell size of $3 \times 3 \mathrm{~cm}^{2}$. The CALICE collaboration has been formed to do research and development of highly granular calorimeters for future lepton colliders. In this proceeding, a quick description of the calorimeter prototypes developed by the CALICE collaboration will be presented in section 2, some highlight results obtained with these prototypes, including tests of Particle Flow Algorithm (PFA) will be the subject of section 3 .

\section{The CALICE calorimeters}

The CALICE calorimeters technologies developed for Particle Flow Algorithm (PFA) calorimeters are displayed in figure 1 left. For all ECAL variants considered, the absorber is tungsten. Its

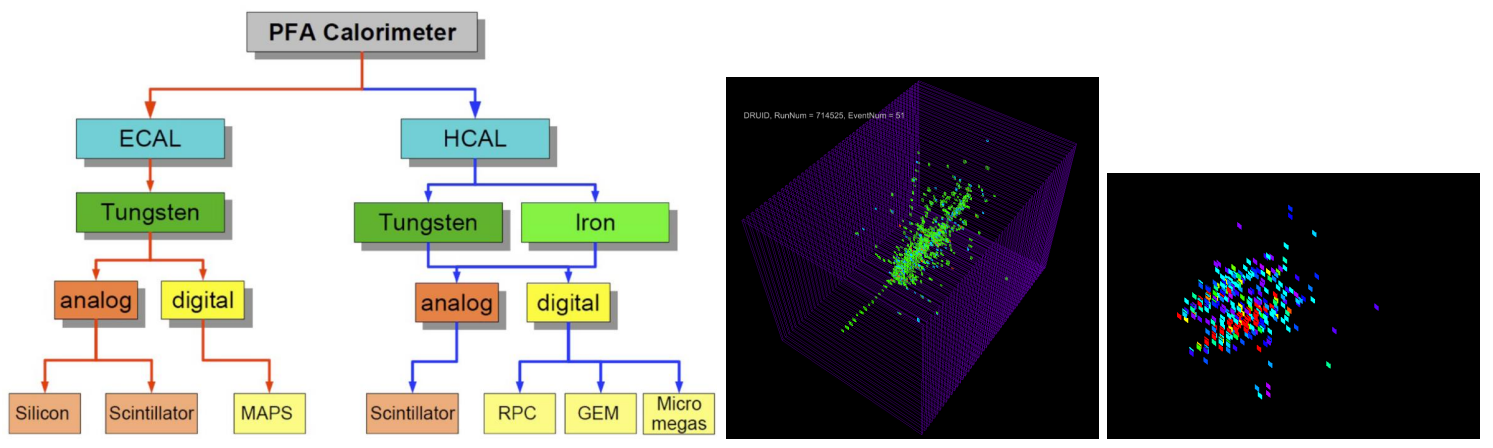

Figure 1: The various highly granular calorimeter technologies developed within the CALICE collaboration (left). Event display of a pion in the SDHCAL[6] (centre) and of two electrons in the SiW-ECAL[7] (right)

small Molière radius $\left(R_{M}=9 \mathrm{~mm}\right)$ and its large ratio of nuclear interaction length over radiation length $\left(\lambda_{I} / X_{0}=28.4\right)$ ensures a good separation between two electromagnetic showers and between electromagnetic and hadronic showers.

\subsection{Physics and technological prototypes}

CALICE calorimeter prototypes are of two kinds. Physics prototypes that demonstrate that high granularity is achievable (the huge number of channels can be coped with) and that the device allows to reconstruct the incoming particle energy. Technological prototypes are updated versions 
of the physics prototypes which are designed to be scalable and that can enter mass production. In particular, all technological prototypes have embedded readout electronics that can store events, analogically or digitally, and have power pulsing capabilities. These requirements on the electronics are to use the planned ILC bunch cycle with collisions occurring during $1 \mathrm{~ms}$ every $200 \mathrm{~ms}[1]$ : record events during collisions, read them out after the $1 \mathrm{~ms}$ bunch train has passed then switch off the electronics until the next bunch train arrives. The fact that the electronics is most of the time switched off reduces the power consumption and the heat produced. No cooling system is then required in the calorimeters, improving their uniformity. All technological prototypes are based on readout chips provided by the Omega group : HARDROC[8], SKIROC[9], SPIROC[10], etc.

\subsection{ECAL prototypes}

Two analogue ECAL prototypes have been designed. One, the ScW-ECAL[11] uses scintillator strips as active medium. The other, the SiW-ECAL[7] uses silicon wafers. Both physics prototypes have similar features : 30 layers, a $1 \times 1 \mathrm{~cm}^{2}$ transverse cell size and an $18 \times 18 \mathrm{~cm}^{2}$ active area. The ScW-ECAL techological prototype is currently built with thinner scintillator strips ( $2 \mathrm{~mm}$ or even $1 \mathrm{~mm}$ thick) read by surface mounted Silicon PhotoMultiplier (SMD-SiPM) and an effective cell size of $5 \times 5 \mathrm{~mm}^{2}$. Ten electronic layers of the SiW-ECAL technological prototypes have been build with a cell size of $5.5 \times 5.5 \mathrm{~mm}^{2}$. A proof of principle for a purely digital ECAL using Monolithic Active Pixel Sensors[12] with a transverse cell size of $50 \times 50 \mu \mathrm{m}^{2}$ has been demonstrated in collaboration with the ALICE Forward Calorimeter[13] development.

\subsection{HCAL prototypes}

A prototype of analogue HCAL (AHCAL)[14] has been produced with tungsten or steel as absorber leading to a depth of $5.3 \lambda_{I}$. The AHCAL physics prototype consists of 38 layers with an active area of $90 \times 90 \mathrm{~cm}^{2}$ made with scintillator tiles read by 16-bit ADCs. The cell size ranges from $3 \times 3 \mathrm{~cm}^{2}$ in the centre up to $12 \times 12 \mathrm{~cm}^{2}$ in the outer regions. A small stack of fifteen $0.13 \mathrm{~m}^{2}$ active layers of the AHCAL technological prototype has been built and tested. A large stack with forty $0.52 \mathrm{~m}^{2}$ layers will be completed by the end of 2017 . The active layers use $3 \times 3 \mathrm{~cm}^{2}$ scintillator tiles with an optimized dome shaped cavity to fit a SMD-SiPM mounted directly on the PCB. Mass production tools for placing the tiles have been developed.

Two gaseous HCAL prototypes, the digital HCAL (DHCAL)[15] and the semi-digital HCAL (SDHCAL)[6] have been built. Both have a $1 \mathrm{~m}^{2}$ active area of Glass Resistive Plate Chambers (GRPC) read by $1 \mathrm{~cm}^{2}$ copper pads interleaved with stainless steel absorber plates. The DHCAL physics prototype has 54 layers for a total depth of $10.47 \lambda_{I}$ and each pad has a binary (1 bit) readout. The SDHCAL technological prototype has 50 layers, $6 \lambda_{I}$, and each pad is read by 3 discriminators ( 3 thresholds, 2 bits readout).

Though the SDHCAL prototype is already technological, further developments are pursued to allow it to be compatible with the proposed ILD HCAL V mechanical structure ${ }^{1}[4][6]$. In this geometry design, active layers have a variable length up to $3 \mathrm{~m}$. Design and construction of length scalable GRPC with their embedded electronics is ongoing, with redesigned gas distribution in the chamber, new daisy-chain PCB with parallel I2C link communication.

\footnotetext{
${ }^{1}$ Also known as the Videau geometry.
} 


\section{Selected highlights of the CALICE calorimeter performance}

\subsection{Constraints on hadron-matter interaction models}

The main strength of the CALICE calorimeters lies in their high granularity which allows, not only to measure the incident particle energy but also to record images of the electromagnetic and hadronic showers (see Figure 1). As an illustration, the SiW-ECAL has been used to study the modelling of pion interactions[16]. A pion interaction in the SiW-ECAL can be tagged from the evolution of the energy deposition per layer. The pion interaction tagging efficiency ranges from $60 \%$ for $2 \mathrm{GeV}$ pion up to $93 \%$ for $10 \mathrm{GeV}$ pion. In this energy range, the probability of a pion interaction is found constant at around $65 \%$, a result compatible with the SiW-ECAL length of $\sim 1 \lambda_{I}$. The energy deposited by the pion after its first interaction has been tagged is proportional to the incoming pion energy (see Figure 2 left), allowing to have a pion energy estimation from a single interaction in the ECAL.

In addition, tracking algorithms have been developed both for the SiW-ECAL[17] and for the SDHCAL using the Hough Transform technique[18]. The characteristics of tracks from pion interactions can then be compared to the model prediction by GEANT4[19] as illustrated in Figure 3 left.
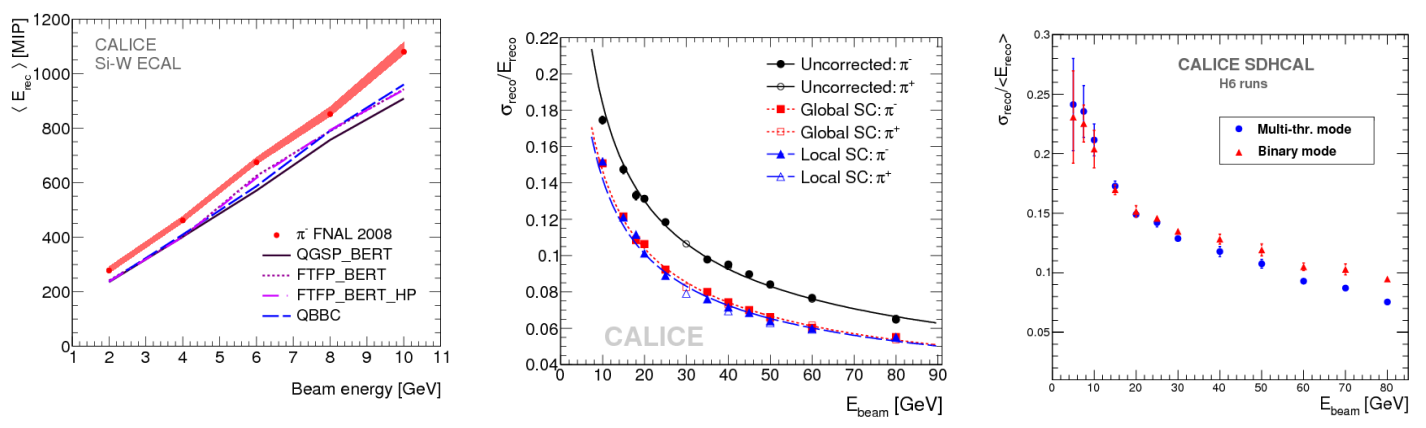

Figure 2: Energy deposited by a pion after its interaction in the SiW-ECAL as a function of the pion incident energy (left), pion energy resolution as a function of the pion energy in the AHCAL with and without software compensation aplied (centre) and in the SDHCAL (right).

\subsection{Hadronic energy reconstruction}

The high granularity of the CALICE HCAL allows to use software based correction to improve the energy reconstruction. In particular, one source of fluctuation is the electromagnetic fraction. Electromagnetic deposits in a shower will tend to have a higher density of deposited energy. Software compensation techniques have been developed for the AHCAL[20] and improves the energy resolution (see Figure 2 centre) by reducing the stochastic term of the resolution from $57.6 \pm 0.4 \%$ down to $44.3 \pm 0.3 \%$. Similar ideas govern the use of semi-digital readout rather than purely binary readout. Fraction of hits recorded above the highest threshold are correlated with the electromagnetic fraction and hence energy reconstruction with multi-threshold mode in SDHCAL improves the energy resolution[21] (see Figure 2 right). 

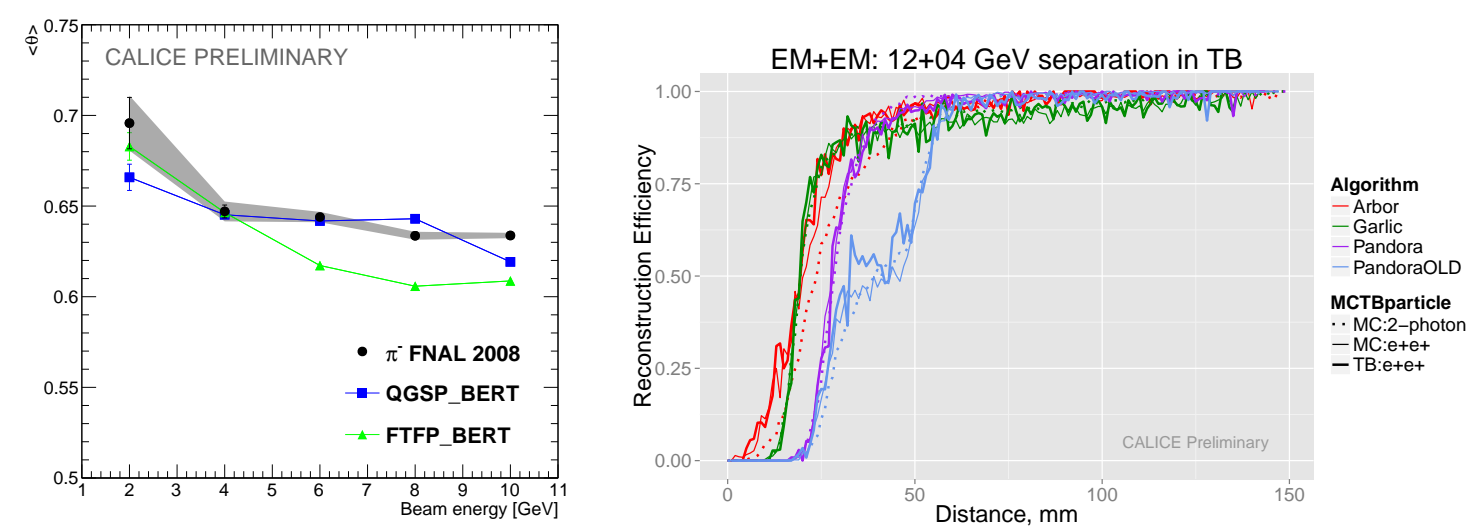

Figure 3: Opening angle distribution of the tracks originating from a pion interaction as a function of the incoming pion energy for pion interacting in the SiW-ECAL (left). Reconstruction efficiency of two electromagnetic showers as a function of the distance between the showers for various Particle Flow Algorithms (right).

\subsection{Particle Flow Algorithms}

CALICE test beam data are used to test PFA separation power. A test sample is formed by combining two single particle showers in one event. In test beam, charged particles are used to emulate a neutral particle by removing the hits from the incoming charged particle before its first interaction. Three algorithms have been tested :

PandoraPFA[22] Most mature, optimized for ILD baseline cell size (SiW-ECAL and AHCAL), designed for analogue device.

ArborPFA[23][24] Use PandoraSDK[25], designed for SDHCAL, small cell size.

GARLIC[26] SiW-ECAL optimised EM shower reconstruction.

Figure 3 right shows the reconstruction efficiency (probability to reconstruct exactly two showers with the energy within $20 \%$ of the true energy and the position within $5 \mathrm{~mm}$ of the true position) for events combining two electromagnetic showers of 12 and $4 \mathrm{GeV}[27]$.

\section{Conclusion}

The CALICE collaboration is developing highly granular calorimeters designed for PFA. The detector concepts are validated with physics or technological prototypes. Tests of particle-matter interaction modelling and of Particle Flow Algorithms are done based on real data. The CALICE technologies are inspiring HL-LHC upgrade. As mentioned earlier, the Digital ECAL uses the same technology as the ALICE FOCAL[13] calorimeter. The CALICE SiW-ECAL is inspiring both the ATLAS HGTD[28] and the ECAL part of the CMS HGCAL[29] while the HCAL part of the HGCAL is inspired by the CALICE AHCAL. 


\section{References}

[1] T. Behnke et al., "The International Linear Collider Technical Design Report - Volume 1: Executive Summary", arXiv:1306.6327 [physics.acc-ph].

[2] CEPC-SPPC Study Group, "CEPC-SPPC Preliminary Conceptual Design Report. 1. Physics and Detector", IHEP-CEPC-DR-2015-01, IHEP-TH-2015-01, IHEP-EP-2015-01.

[3] J. C. Brient, J. Phys. Conf. Ser. 160 (2009) 012025. doi:10.1088/1742-6596/160/1/012025

[4] T. Behnke et al., "The International Linear Collider Technical Design Report - Volume 4: Detectors", arXiv:1306.6329 [physics.ins-det].

[5] J. C. Brient, "Improving the jet reconstruction with the particle flow method: An introduction", INSPIRE-672270

[6] G. Baulieu et al., "Construction and commissioning of a technological prototype of a high-granularity semi-digital hadronic calorimeter", JINST 10 (2015) no.10, P10039 doi:10.1088/1748-0221/10/10/P10039 [arXiv:1506.05316 [physics.ins-det]].

[7] J. Repond et al. [CALICE Collaboration], "Design and Electronics Commissioning of the Physics Prototype of a Si-W Electromagnetic Calorimeter for the International Linear Collider", JINST 3 (2008) P08001 [arXiv:0805.4833 [physics.ins-det]].

[8] F. Dulucq, C. de La Taille, G. Martin-Chassard and N. Seguin-Moreau, "HARDROC: Readout chip for CALICE/EUDET Digital Hadronic Calorimeter", doi:10.1109/NSSMIC.2010.5874060

[9] M. Bouchel et al. [CALICE and EUDET Collaborations], "Skiroc: A front-end chip to read out the imaging silicon-tungsten calorimeter for ILC", CERN-2008-008.

[10] S. Conforti Di Lorenzo, S. Callier, J. Fleury, F. Dulucq, C. De la Taille, G. M. Chassard, L. Raux and N. Seguin-Moreau, "SPIROC: Design and performances of a dedicated very front-end electronics for an ILC Analog Hadronic CALorimeter (AHCAL) prototype with SiPM read-out", JINST 8 (2013) C01027. doi:10.1088/1748-0221/8/01/C01027

[11] K. Francis et al. [CALICE Collaboration], "Performance of the first prototype of the CALICE scintillator strip electromagnetic calorimeter", Nucl. Instrum. Meth. A 763 (2014) 278 doi:10.1016/j.nima.2014.06.039 [arXiv:1311.3761 [physics.ins-det]].

[12] J. A. Ballin et al., "TPAC: A 0.18 micron MAPS for digital electromagnetic calorimetry at the ILC", doi:10.1109/NSSMIC.2008.4774795

[13] C. Zhang [ALICE FoCal Group], "FoCal - A high granularity electromagnetic calorimeter for forward direct photon measurements", Nucl. Instrum. Meth. A 845 (2017) 542. doi:10.1016/j.nima.2016.06.075

[14] C. Adloff et al. [CALICE Collaboration], "Construction and Commissioning of the CALICE Analog Hadron Calorimeter Prototype", JINST 5 (2010) P05004 doi:10.1088/1748-0221/5/05/P05004 [arXiv:1003.2662 [physics.ins-det]].

[15] C. Adams et al., "Design, construction and commissioning of the Digital Hadron Calorimeter-DHCAL", JINST 11 (2016) no.07, P07007 doi:10.1088/1748-0221/11/07/P07007 [arXiv:1603.01653 [physics.ins-det]].

[16] B. Bilki et al. [CALICE Collaboration], "Testing hadronic interaction models using a highly granular silicon-tungsten calorimeter", Nucl. Instrum. Meth. A 794 (2015) 240 doi:10.1016/j.nima.2015.05.009 [arXiv:1411.7215 [physics.ins-det]]. 
[17] The CALICE collaboration, "Tracks of hadronic showers in the Si-W ECAL physics prototype", CALICE Analysis Note-055

[18] Z. Deng et al. [CALICE Collaboration], "Tracking within Hadronic Showers in the CALICE SDHCAL prototype using a Hough Transform Technique", JINST 12 (2017) no.05, P05009 doi:10.1088/1748-0221/12/05/P05009 [arXiv:1702.08082 [physics.ins-det]].

[19] S. Agostinelli et al. [GEANT4 Collaboration], "GEANT4: A Simulation toolkit", Nucl. Instrum. Meth. A 506 (2003) 250. doi:10.1016/S0168-9002(03)01368-8

[20] C. Adloff et al. [CALICE Collaboration], "Hadronic energy resolution of a highly granular scintillator-steel hadron calorimeter using software compensation techniques", JINST 7 (2012) P09017 doi:10.1088/1748-0221/7/09/P09017 [arXiv:1207.4210 [physics.ins-det]].

[21] V. Buridon et al. [CALICE Collaboration], "First results of the CALICE SDHCAL technological prototype", JINST 11 (2016) no.04, P04001 doi:10.1088/1748-0221/11/04/P04001 [arXiv:1602.02276 [physics.ins-det]].

[22] J. S. Marshall and M. A. Thomson, "Pandora Particle Flow Algorithm", arXiv:1308.4537 [physics.ins-det].

[23] M. Ruan and H. Videau, "Arbor, a new approach of the Particle Flow Algorithm", arXiv:1403.4784 [physics.ins-det].

[24] The CALICE collaboration, "Separation of nearby hadronic showers in the CALICE SDHCAL prototype detector using ArborPFA", CALICE Analysis Note-054

[25] J. S. Marshall and M. A. Thomson, "The Pandora software development kit for particle flow calorimetry", J. Phys. Conf. Ser. 396 (2012) 022034. doi:10.1088/1742-6596/396/2/022034

[26] D. Jeans, J. C. Brient and M. Reinhard, "GARLIC: GAmma Reconstruction at a LInear Collider experiment", JINST 7 (2012) P06003 doi:10.1088/1748-0221/7/06/P06003 [arXiv:1203.0774 [physics.ins-det]].

[27] The CALICE collaboration, "Separation of two overlapped electromagnetic or electromagnetic-hadronic showers in CALICE highly granular physics calorimeter prototypes using Pandora, Garlic and Arbor Particle Flow Algorithms", CALICE Analysis Note-057

[28] ATLAS collaboration, "ATLAS Phase-II Upgrade Scoping Document", CERN-LHCC-2015-020

[29] A. M. Magnan [CMS Collaboration], "HGCAL: a High-Granularity Calorimeter for the endcaps of CMS at HL-LHC", JINST 12 (2017) no.01, C01042. doi:10.1088/1748-0221/12/01/C01042 